\title{
Pure risk premiums under deductibles ${ }^{1}$
}

\author{
Krzysztof Burnecki, Joanna Nowicka-Zagrajek and Aleksander Weron
}

\author{
Hugo Steinhaus Center for Stochastic Methods, \\ Institute of Mathematics, Wrocław University of Technology, \\ Wyspiańskiego 27, 50-370 Wrocław
}

\section{Introduction}

The choice of a deductible which will be incorporated in the contract and the right pricing of premium under the deductible is vital for the insurance company. The pricing is crucial since too low a price level results in a loss, while with too high rates a company can price itself out of the market. It is the actuary's task to find methods of premium calculation that take into account possible deductibles. In the paper we present new results which can assist in solving these real-world management problems.

The idea of a deductible is, firstly, to reduce claim handling costs by excluding coverage for the often numerous small claims and, secondly, to provide some motivation to the insured to prevent claims, through a limited degree of participation in claim costs (see e.g. [3] and [6]). If we want to describe in detail the reasons for introducing deductibles we have to mention the following properties of a deductible:

(i) loss prevention - as the compensation is reduced by a deductible the retention of the insured is positive. This makes out a good case for avoiding the loss.

(ii) loss reduction - the fact that the deductible puts the policyholder at risk of obtaining only partial compensation provides an economic incentive to reduce the extent of the damage.

(iii) avoidance of small claims where administration costs are dominant - for small losses, the administration costs will often exceed the loss itself, and hence the insurance company would want the policyholder to pay it himself.

(iv) premium reduction - premium reduction can be an important aspect for the policyholders, they may prefer to take a higher deductible to get a lower premium.

\footnotetext{
${ }^{1}$ Research supported in part by KBN Grant No. PBZ KBN 016/P03/1999.
} 
In Section 2 we derive formulae for pure risk premiums under franchise, fix amount, proportional, limited proportional and disappearing deductibles in terms of the limited expected value function. In Section 3 we consider a loss data of Pumped Storage Power Plants Co. (see [7]). We discuss pure risk premiums under the deductibles for the wellknown lognormal loss distribution. We illustrate graphically the influence of the parameters of the discussed deductibles on the pure risk premium.

\section{General formulae for premiums under deductibles}

Let $X$ be the total (random) monetary amount of some economic risk; we will briefly call $X$ a risk. A premium calculation principle is a rule saying what premium should be assigned to a given risk. In the paper we will apply the simplest premium (calculation principle) which is called pure risk premium, namely the mean of $X$. It is often applied in life and some mass lines of business in non-life insurance (see [5]). As we know from ruin theory, the pure risk premium without any kind of loading is insufficient since, in the long run, ruin is inevitable even in the case of substantial (though finite) initial reserves. Nevertheless, the pure risk premium can be - and still is - of practical use because, for one thing, in practice the planning horizon is always limited, and for another, because there are indirect ways of loading a premium, e.g. by neglecting interest earnings.

More precisely, let $X$ denote a non-negative random variable describing the size of claim (risk, loss), $F(t)$ and $f(t)$ its distribution and probability distribution functions respectively, and $h(x)$ the payment function corresponding to a deductible. Moreover, we assume that the expected value EX exists. The pure risk premium $P$ (as we consider only pure risk premiums we will henceforth use the term 'premium' meaning 'pure risk premium') is then equal to the expectation, i.e. $P=\operatorname{Eh}(X)$.

In the case of no deductible the payment function is obviously of the form $h(x)=x$. This means that if the loss is equal to $X$, the insurer pays the whole claim amount and $P=$ $\mathrm{E} X$.

In order to derive formulae for premiums under deductibles let us recall the socalled limited expected value function, namely

$$
E(X, x)=\int_{0}^{x} y f(y) d y+x(1-F(x)), \quad x>0
$$


The value of this function at a point $X$ is equal to the expected value of the random variable $X$ truncated at the point $X$. The function is a very useful tool for testing the goodness of fit of an analytic distribution function to the observed claim size distribution function, see e.g. [3].

In the following subsections we will consider the most important types of deductibles and derive corresponding premium formulae.

\subsection{Franchise deductible}

One of the deductibles that can be incorporated in the contract is a so-called franchise deductible. In this case the insurer pays the whole claim, if the agreed deductible amount is exceeded. More precisely, under the franchise deductible of $a$, if the loss is less than $a$ amount the insurer pays nothing, but if the loss equals or exceeds $a$ amount claim is paid in full. This means that the payment function can be described by

$$
h_{F D(a)}=\left\{\begin{array}{lc}
0, & x<a \\
x, & \text { otherwise } .
\end{array}\right.
$$

It is worth noticing that the franchise deductible satisfies properties (i), (iii) and (iv), but not property (ii). This deductible can even work against property (ii) - since if a loss occurs, the policyholder would prefer it to be greater than or equal to the deductible.

The pure risk premium under the franchise deductible can be expressed in terms of the premium in the case of no deductible and the corresponding limited expected value function.

Formula 1. The pure risk premium in the case of the franchise deductible of $a$ is given by

$$
P_{F D(a)}=P-\mathrm{E}(X, a)+a(1-F(a))
$$

It can be easily noticed that this premium is a decreasing function of $a$, when $a=0$ the premium is equal to the one in the case of no deductible and if $a$ tends to infinity the premium tends to zero.

\subsection{Fixed amount deductible}

An agreement between the insured and the insurer incorporating a deductible $b$ means that the insurer pays only the part of the claim which exceeds the amount $b$; if the size of the claim falls below this amount, then the claim is not covered by the contract and the insured receives no indemnification. The payment function is thus given by

$$
h_{F A D(b)}(x)=\max (0, x-b) \text {. }
$$


The fixed amount deductible satisfies all properties mentioned in Section 1. The following formula expresses the premium in the case of fixed amount deductible in terms of the premium under the franchise deductible.

Formula 2. The pure risk premium in the case of the fixed amount deductible of $b$ is given by

$$
P_{F A D(b)}=P-\mathrm{E}(X, b)=P_{F D(b)}-b(1-F(b)) .
$$

As previously, the premium is a decreasing function of $b$, for $b=0$ it gives the premium in the case of no deductible and if $b$ tends to infinity, it tends to zero.

\subsection{Proportional deductible}

In the case of the proportional deductible of $c$, where $c \in(0,1)$, each payment is reduced by $c \cdot 100 \%$ (the insurer pays $(1-c) \cdot 100 \%$ of the claim). Consequently, the payment function is given by

$$
h_{P D(c)}(x)=(1-c) x .
$$

The proportional deductible satisfies properties (i), (ii) and (iv), but not property (iii), as it implies some compensation for even very small claims. The following formula shows a simple relation between the premium under proportional deductible and the premium in the case of no deductible.

Formula 3. The pure risk premium in the case of the proportional deductible of $c$, where $c \in(0,1)$, is given by

$$
P_{P D(c)}=(1-c) P \text {. }
$$

Clearly, the premium is a decreasing function of $c, P_{P D(0)}=P$ and $P_{P D(1)}=0$.

\subsection{Limited proportional deductible}

The proportional deductible is usually combined with a minimum amount deductible so the insurer does not need to handle small claims and with maximum amount deductible to limit the retention of the insured. For the proportional deductible of $c$ with minimum amount $m_{1}$ and maximum amount $m_{2}\left(m_{1}<m_{2}\right)$ the payment function is given by

$$
h_{L P D\left(c, m_{1}, m_{2}\right)}(x)=\left\{\begin{array}{cc}
0, & x \leq m_{1} \\
x-m_{1}, & m_{1}<x \leq m_{1} / c \\
(1-c) x, & m_{1} / c<x \leq m_{2} / c \\
x-m_{2}, & \text { otherwise. }
\end{array}\right.
$$

The limited proportional deductible satisfies all properties mentioned in Section 1. The following formula expresses the premium under the limited proportional deductible in 
terms of the premium in the case of no deductible and the corresponding limited expected value function.

Formula 4. The pure risk premium in the case of the limited proportional deductible of $c$ with minimum amount $m_{1}$ and maximum amount $m_{2}\left(m_{1}<m_{2}\right)$ is given by

$$
P_{L P D(c, m 1, m 2)}=P-\mathrm{E}\left(X, m_{1}\right)+c\left[\mathrm{E}\left(X, m_{1} / c\right)-\mathrm{E}\left(X, m_{2} / c\right)\right] .
$$

Sometimes only one limitation is incorporated in the contract, i.e. $m_{1}=0$ or $m_{2}=\infty$. It is easy to check that the limited proportional deductible with $m_{1}=0$ and $m_{2}=\infty$ reduces to the proportional deductible.

\subsection{Disappearing deductible}

In the case of the disappearing deductible the payment depends on the loss in the following way: if the loss is less than an amount of $d_{1}$, the insurer pays nothing, if the loss exceeds $d_{2}$ $\left(d_{2}>d_{1}\right)$ amount, the insurer pays the loss in full, if the loss is between $d_{1}$ and $d_{2}$, then the deducible is reduced linearly between $d_{1}$ and $d_{2}$. The payment function is thus given by

$$
h_{D D\left(d_{1}, d_{2}\right)}(x)=\left\{\begin{array}{cc}
0, & x \leq d_{1} \\
\frac{d_{2}\left(x-d_{1}\right)}{d_{2}-d_{1}}, & d_{1}<x \leq d_{2}, \\
x, & \text { otherwise } .
\end{array}\right.
$$

This kind of deductible satisfies properties (i), (iii) and (iv), but similarly as in the case of the franchise deductible it works against (ii). The following formula shows the premium under the disappearing deductible in terms of the premium in the case of no deductible and the corresponding limited expected value function.

Formula 4. The pure risk premium in the case of the disappearing deductible of $d_{1}$ and $d_{2}$ $\left(d_{1}<d_{2}\right)$ is given by

$$
P_{D D(d 1, d 2)}=P+\mathrm{d}_{1} /\left(d_{2}-d_{1}\right\} \mathrm{E}\left(X, d_{2}\right)-\mathrm{d}_{2} /\left(d_{2}-d_{1}\right\} \mathrm{E}\left(X, d_{1}\right) .
$$

If $d_{1}=0$, then the premium does not depend on $d_{2}$ and it becomes the premium in the case of no deductible. If $d_{2}$ tends to infinity, then the disappearing deductible reduces to the fix amount deductible of $d_{1}$.

\section{Example}

The derivation of claim size distributions from the claim data could be considered to be a separate discipline in its own right, which requires applying methods of mathematical 
statistics, cf. [3]. The objective is to find a distribution function $F$ which fits the observed data in a satisfactory manner. The approach most frequently adopted in the actuarial literature is to find a suitable analytic expression which fits the observed data well and which is easy to handle, see e.g. [1].

The lognormal distribution is a typical candidate when looking for a suitable analytic distribution which fits the observed data well, see e.g. [1] and [4]. Consider a random variable $Z$ which has the normal distribution. Let $X=\exp (Z)$. The distribution of $X$ is called a lognormal distribution. The distribution function is given by

$$
F(t)=\Phi\left(\frac{\ln t-\mu}{\sigma}\right)=\int_{0}^{t} \frac{1}{\sqrt{2 \pi} \sigma y} \exp \left\{-\frac{1}{2}\left(\frac{\ln y-\mu}{\sigma}\right)^{2}\right\} d y, \quad t, \sigma>0, \mu \in R,
$$

where $\Phi(\cdot)$ is the standard normal (with mean 0 and variance 1 ) distribution function. The lognormal distribution is very useful in modelling of claim costs. It has a thick right tail and fits many situations well.

We conducted in [7] empirical studies for the losses occurred in Pumped Storage Power Plants (PSPP) Co. in Poland between 1991 and 1999. Different distributions were fitted using the moments and maximum likelihood estimation. The lognormal distribution passed all tests and we now choose it for the illustration.

Example 1. For the lognormal distribution the following formulae hold (see [2]):

- franchise deductible premium $P_{F D(a)}=\exp \left(\mu+\sigma^{2} / 2\right)\left(1-\Phi\left(\frac{\ln a-\mu-\sigma^{2}}{\sigma}\right)\right)$,

- fixed amount deductible premium

$$
P_{F D(a)}=\exp \left(\mu+\sigma^{2} / 2\right)\left(1-\Phi\left(\frac{\ln b-\mu-\sigma^{2}}{\sigma}\right)\right)-b\left(1-\Phi\left(\frac{\ln b-\mu}{\sigma}\right)\right),
$$

- $\quad$ proportional deductible premium $P_{P D(c)}=(1-c) \exp \left(\mu+\sigma^{2} / 2\right)$,

- limited proportional deductible premium

$$
\begin{aligned}
& P_{F D(a)}=\exp \left(\mu+\sigma^{2} / 2\right)\left(1-\Phi\left(\frac{\ln m_{1}-\mu-\sigma^{2}}{\sigma}\right)\right)+m_{1}\left(\Phi\left(\frac{\ln m_{1}-\mu}{\sigma}\right)-\Phi\left(\frac{\ln \left(m_{1} / c\right)-\mu}{\sigma}\right)\right) \\
& +c \exp \left(\mu+\sigma^{2} / 2\right)\left(\Phi\left(\frac{\ln \left(m_{1} / c\right)-\mu-\sigma^{2}}{\sigma}\right)-\Phi\left(\frac{\ln \left(m_{2} / c\right)-\mu-\sigma^{2}}{\sigma}\right)\right) \\
& +m_{2}\left(\Phi\left(\frac{\ln \left(m_{2} / c\right)-\mu}{\sigma}\right)-1\right),
\end{aligned}
$$


- disappearing deductible premium

$$
\begin{aligned}
& P_{F D(a)}=\exp \left(\mu+\sigma^{2} / 2\right) /\left(d_{2}-d_{1}\right)\left(d_{2}-d_{1}+d_{1} \Phi\left(\frac{\ln d_{2}-\mu-\sigma^{2}}{\sigma}\right)-d_{2} \Phi\left(\frac{\ln d_{1}-\mu-\sigma^{2}}{\sigma}\right)\right) \\
& +d_{1} d_{2} /\left(d_{2}-d_{1}\right)\left(\Phi\left(\frac{\ln d_{1}-\mu}{\sigma}\right)-\Phi\left(\frac{\ln d_{2}-\mu}{\sigma}\right)\right) .
\end{aligned}
$$

The premium under no deductible corresponding to the lognormal distribution is nearly PLN 2.3 million. Now, we are going to show the influence of incorporating different deductibles on the premium. Let us first concentrate on franchise and fixed amount deductibles. Figure 1 depicts the comparison of the two corresponding premiums and the effect of increasing the deductible $a$ and $b$. Evidently $P \geq P_{F D} \geq P_{F A D}$. Moreover, we can see that deducible of about PLN 10 million in the lognormal case reduces $P_{F A D}$ by half.

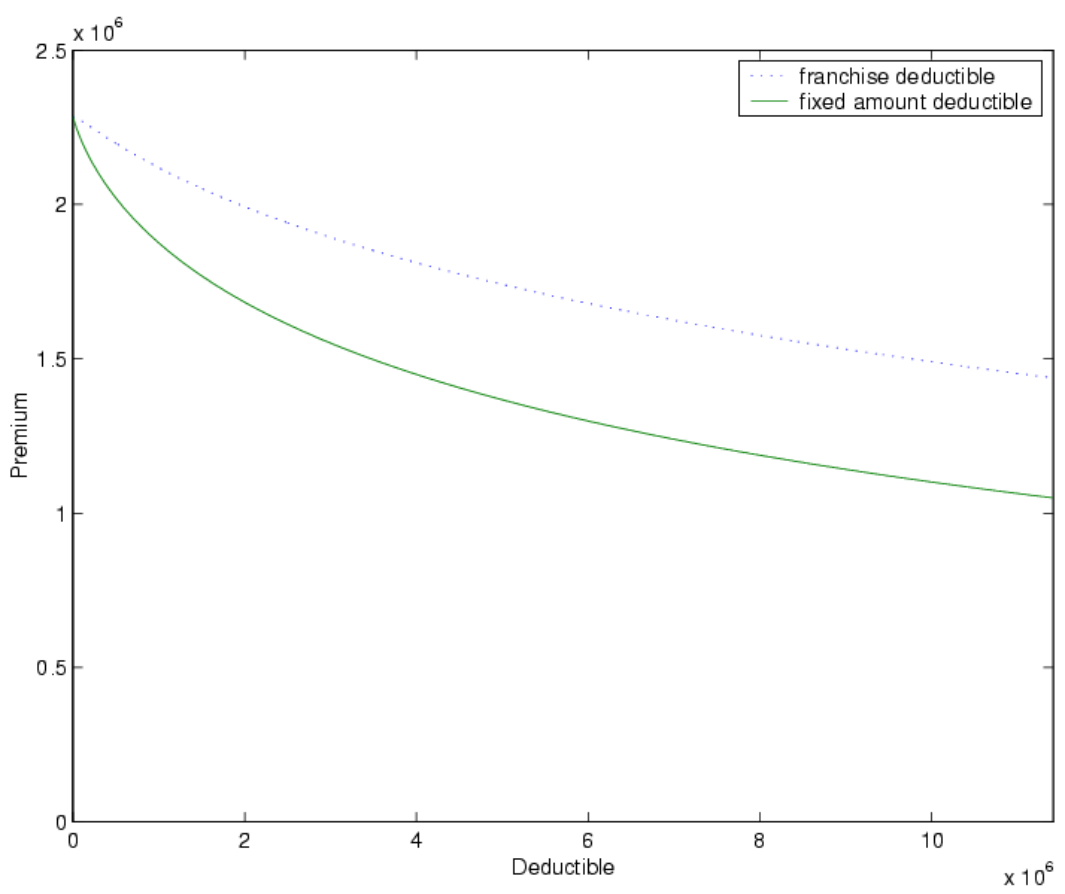

Figure 1. The premium under franchise and fixed amount deductibles. The lognormal case based on the PSPP Co. loss data.

The proportional deductible influences the premium in an obvious manner, that is pro rata (e.g. $c=0.25$ results in cutting the premium by a quarter). Figure 2 depicts the effect of parameters $c, m_{1}$ and $m_{2}$ of the limited proportional deductible. It is easy to see that $P_{L P D(c, m 1, m 2)}$ is a decreasing function of these parameters. 


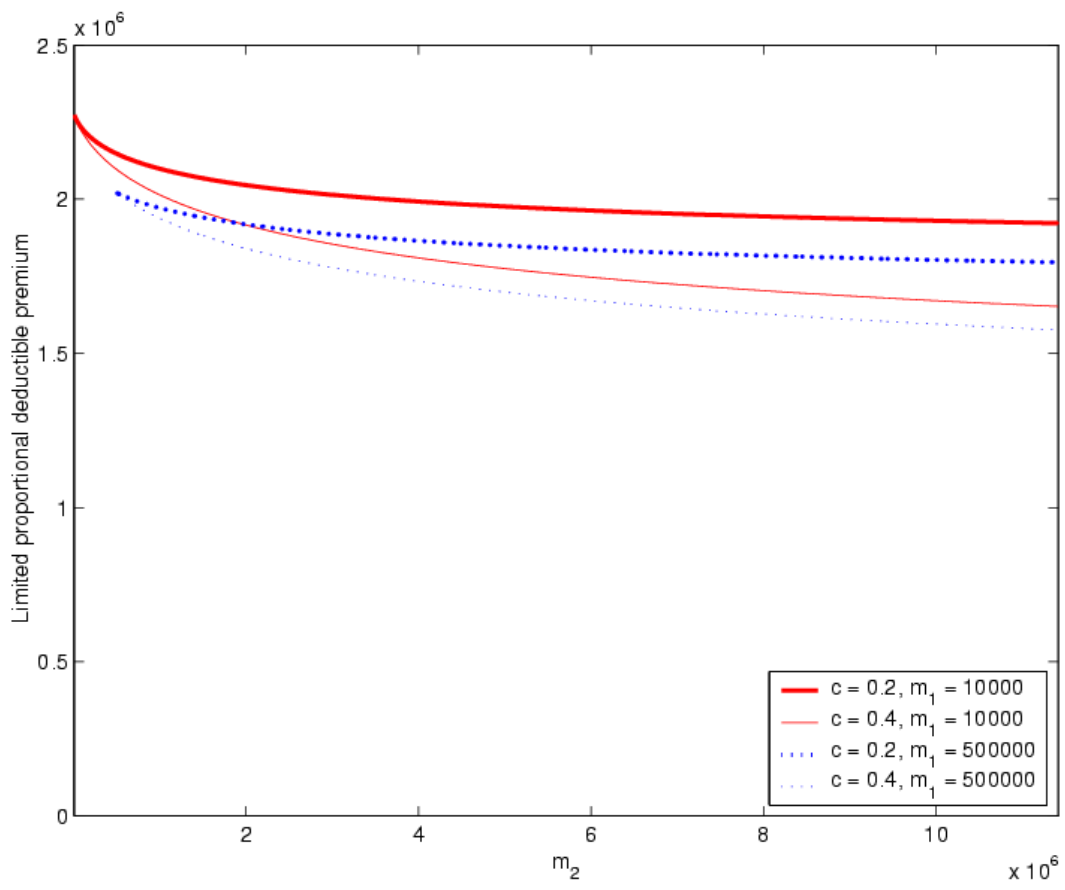

Figure 2. The premium under the limited proportional deductible. The lognormal case based on the PSPP Co. loss data.

Finally, Figure 3 depicts the influence of parameters $d_{1}$ and $d_{2}$ of the disappearing deductible. Clearly, $P_{D D(d 1, d 2)}$ is a decreasing function of the parameters and we can observe that the effect of increasing $d_{2}$ is rather minor.

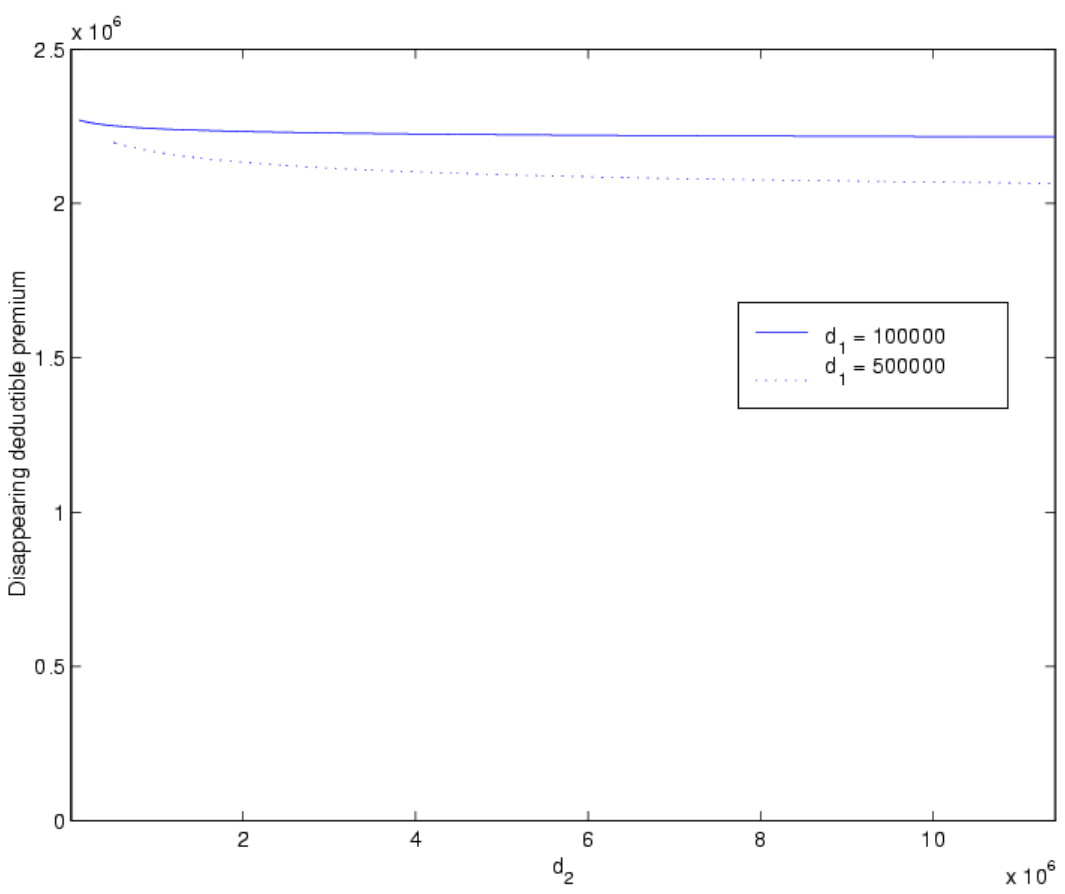

Figure 3. The premium under the disappearing deductible. The lognormal case based on the PSPP Co. loss data. 


\section{References}

[1] Burnecki, K., Kukla, G. and Weron, R. (2000). Property insurance loss distributions. Physica A, Vol. 287, $269-278$.

[2] Burnecki, K., Nowicka-Zagrajek, J. and Wyłomańska, A. (2004). Pure risk premiums under deductibles, in Cižek, P., Härdle, W., Weron, R., eds., Statistical Tools for Finance and Insurance, Springer, Heidelberg.

[3] Daykin, C.D., Pentikäinen, T. and Pesonen, M. (1994). Practical Risk Theory for Actuaries, Chapman \& Hall, London.

[4] Embrechts, P., Klüppelberg and C., Mikosch, T. (1997). Modelling Extremal Events, Springer, Berlin.

[5] Straub, E. (1988). Non-Life Insurance Mathematics, Springer, Berlin.

[6] Sundt, B. (1993). An Introduction to Non-Life Insurance Mathematics, 3rd edition, Verlag Versicherungswirtschaft e.V., Karlsruhe.

[7] Weron, A. et al. (2000). Optimal insurance strategy based on breakdown and loss analysis of PSPP Co. property. Technical Report, Wrocław. 